Int. J. Contemp. Math. Sciences, Vol. 2, 2007, no. 19, 929 - 942

\title{
Bounds for Recurrences on Ranked Posets
}

\author{
Kenneth S. Berenhaut ${ }^{1} 2$ \\ Department of Mathematics \\ Wake Forest University \\ Winston-Salem, NC, 27109, USA \\ berenhks@wfu.edu \\ Augustine B. O'Keefe \\ Department of Mathematics \\ Wake Forest University \\ Winston-Salem, NC, 27109, USA \\ okeeab4@wfu.edu \\ Filip Saidak \\ Department of Mathematics \\ University of North Carolina at Greensboro \\ Winston-Salem, NC, 27109, USA \\ f_saidak@uncg.edu
}

\begin{abstract}
This note considers an extension of the concept of linear recurrence to recurrences on ranked posets. Some results on growth rates in the linear case are then extended to this generalized scenario. The work is motivated by recent results on multi-dimensional recurrences which have had applications for obtaining bounds for complex multidimensional generating functions. Some further connections to Möbius functions for binary relations and inverses of $\{0,1\}$ triangular matrices are also discussed.
\end{abstract}

Mathematics Subject Classification: 39A10, 06A07, 05D99, 11B37, 39A11, 15A36

Keywords: Explicit bounds, Recurrences, Ranked posets, Directed graphs, Möbius functions, Level graphs, Difference equations, Growth rates, Nonconstant coefficients, $\{0,1\}$ matrices

\footnotetext{
${ }^{1}$ Corresponding author.

${ }^{2}$ The author acknowledges financial support from a Sterge Faculty Fellowship.
} 


\section{Introduction}

This paper studies an extension of the concept of linear recurrence to recurrences on ranked and graded partially ordered sets. In particular, a poset $P=(X,<)$ is ranked if there exists a ranking function $r: P \rightarrow \mathbb{N}$ such that $r(x)=r(y)+1$ whenever $x$ covers $y$, and a ranked poset is graded if all maximal chains have equal length. For further discussion of posets and associated terminology see, for instance, [18].

For convenience, we include here some additional standard definitions. Firstly, an antichain in $P$ is a set of pairwise incomparable elements. The size of the longest antichain in $P$ is referred to as the partial order width $w(P)$. We also denote the number of elements in $P$ by $\|P\|$.

We are particularly interested here in ordinal sums of antichains (also known as layered posets). Suppose $Q=\left(Y_{1},<\right)$ and $R=\left(Y_{2},<\right)$ are posets with disjoint underlying sets $Y_{1}$ and $Y_{2}$, respectively. Their ordinal sum, $Q \oplus R$, is defined to be the poset with underlying set $Y_{1} \cup Y_{2}$, with $x<y$ if and only if $x<y$ in $P$, or $x<y$ in $Q$, or $x \in Y_{1}$ and $y \in Y_{2}$, while, the union (or direct sum) of $Q$ and $R, Q+R$, is the poset with underlying set $Y_{1} \cup Y_{2}$, with $x<y$ if and only if $x<y$ in $P$, or $x<y$ in $Q$.

In particular, we have that the finite chain with $n$ elements can be written as $\boldsymbol{n}=\mathbf{1} \oplus \cdots \oplus \mathbf{1}$ and the $n$ element antichain is a sum $n \mathbf{1}=\mathbf{1}+\cdots+\mathbf{1}$. The posets of interest here will also be assumed to be bounded with unique maximal element $\hat{1}$ and unique minimal element $\hat{0}$. Hence we will be considering posets $P$ of the form

$$
P=\hat{0} \oplus l_{1} \mathbf{1} \oplus l_{2} \mathbf{1} \oplus \cdots \oplus l_{n} \mathbf{1} \oplus \hat{1}
$$

for some sequence of positive integers $\left\{l_{i}\right\}$. Here $l_{i}=\left\|V_{i}\right\|$, where $V_{i}=\{x$ : $r(x)=i\}$ for $i \geq 0$ with $r$ the given ranking function for $P$ and $r(\hat{0})$ taken to be zero.

In terms of associated Hasse diagrams, we are restricting attention to graphs $G$ with a unidirectional flow, $n$ levels and completeness between adjacent levels: $G=(V, E)$ with vertex set $V=V_{0} \cup V_{1} \cup \cdots \cup V_{k}$ such that $V_{i} \cap V_{j}=\emptyset$, for every $0 \leq i<j \leq k$ (with $\left\|V_{0}\right\|=\left\|V_{k}\right\|=1$ ) and edge set $E=\left\{x y \mid x \in V_{i}, y \in V_{i+1}, 0 \leq i<k\right\}$. A simple graph of this type is a line with $V_{i}=\left\{x_{i}\right\}$ and $E=\left\{x_{0} x_{1}, x_{1} x_{2}, x_{2} x_{3}, \cdots, x_{i-1} x_{i}, \cdots, x_{n-1} x_{n}\right\}$. In this case the recurrences of interest will reduce to the heavily studied simple linear recursive sequences (c.f. [9]).

Recently, in [3], recurrences on multidimensional lattices were considered in order to examine zero-free regions for multidimensional complex power series. In the two dimensional case, the problem there can be expressed in terms of recurrences for graphs where the levels are left to right diagonals in the upper right half plane (see Figure 1). For further discussion of multi-variable 
generating functions and related multivariate linear recurrences see [15], [16], [17], and the references therein.
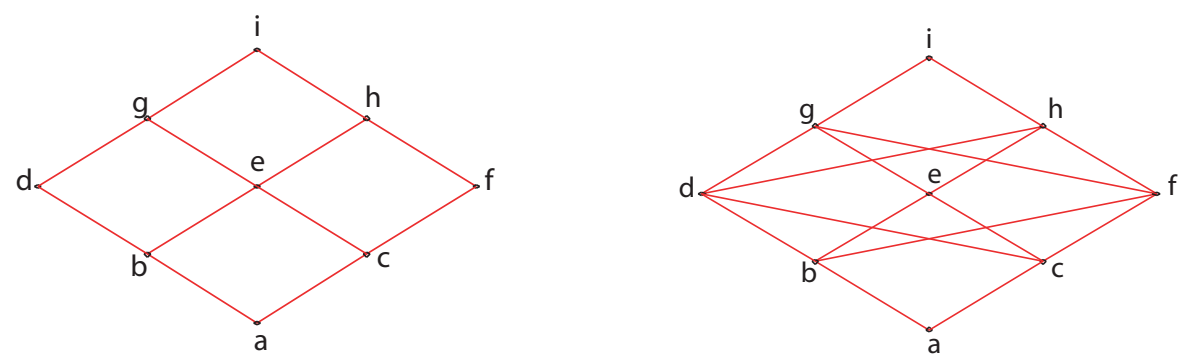

Figure 1: A comparison of a Hasse diagram of the sort considered in [3] with that for the associated ordinal sum.

To define a recurrence on a ranked and graded poset $P$ (with ranking function $r$ ) of $\operatorname{order} \kappa$, we consider a function $f$ on $P$ satisfying, for $x \in P$,

$$
f(x)=\sum_{\substack{y<x \\ 1 \leq r(x)-r(y) \leq \kappa}} \alpha_{x, y} f(y)
$$

for some double sequence $\left\{\alpha_{u, v}\right\}$.

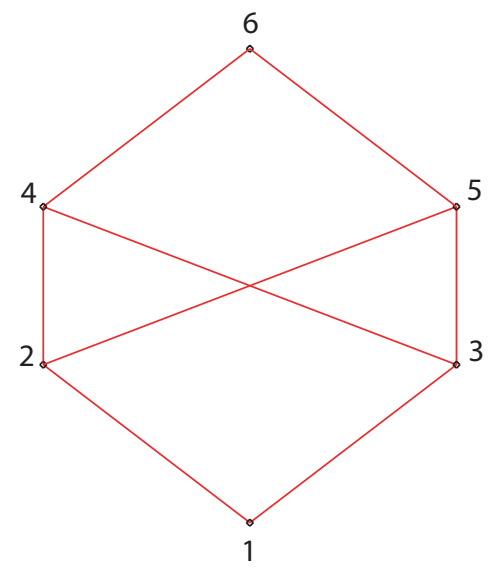

Figure 2: Hasse diagram for the poset of Example 1.

Example 1. Consider the poset for which the Hasse diagram is given in Figure 2, and assign $\alpha_{u, v}$ for $u \leq v$ according to the following matrix. 


$$
\left[\begin{array}{lllll}
\alpha_{2,1} & & & & \\
\alpha_{3,1} & & & & \\
\alpha_{4,1} & \alpha_{4,2} & \alpha_{4,3} & & \\
\alpha_{5,1} & \alpha_{5,2} & \alpha_{5,3} & & \\
\alpha_{6,1} & \alpha_{6,2} & \alpha_{6,3} & \alpha_{6,4} & \alpha_{6,5}
\end{array}\right]=\left[\begin{array}{llllll}
-0.5 & & & & \\
-0.2 & & & & \\
-0.3 & -1.0 & -1.0 & & \\
-0.2 & -0.5 & -0.8 & & \\
-0.7 & -0.1 & -0.3 & -1.0 & -0.5
\end{array}\right] .
$$

For $\kappa \geq 3$, we then have from $(2)$, that $f(1)=1, f(2)=-0.5(1)=-0.5$, $f(3)=-0.2(1)=-0.2, f(4)=-0.3(1)+(-1.0)(-0.5)+(-1.0)(-0.2)=0.4$, $f(5)=-0.2(1)+(-0.5)(-0.5)+(-0.8)(-0.2)=0.21$ and $f(6)=-0.7(1)+$ $(-0.1)(-0.5)+(-0.3)(-0.2)+(-1.0)(0.4)+(-0.5)(0.21)=-1.095$.

We now turn to the statement of our results and some applications.

\section{Statement of Results}

It is known, (c.f. the arguments in [5], [6], [14] and [11]) that for $\kappa=\infty$, in the linear (finite chain) case, if $f(\hat{0})=1$, and $\alpha_{u, v} \in[-1,0]$, for all $u, v \in P$, then for $i \geq 1$

$$
\left|f\left(x_{i}\right)\right| \leq F_{i}
$$

where $F_{i}$ is the $i^{\text {th }}$ Fibonacci number. For related results for power series with restricted coefficients see also [10], [12] and [20]. The question was further investigated for odd $\kappa$ in [2]. In this initial foray into the question of bounding recurrences on partially ordered sets, we prove the following natural (though nontrivial) extension.

Theorem 1 Suppose that a poset $P$ satisfies (1) for a sequence $\left\{l_{i}\right\}$ of positive integers. If $\kappa=\infty, f(\hat{0})=1$, and

$$
\alpha_{u, v} \in[-1,0]
$$

for all $u, v \in P$, then for $x \in P$ with $r(x)=i \geq 1$, we have

$$
|f(x)| \leq B_{i}
$$

where $\left\{B_{i}\right\}$ is defined by $B_{0}=0, B_{1}=1$ and

$$
B_{i}=l_{i-1} B_{i-1}+B_{i-2},
$$

for $i \geq 2$. 
Note that Theorem 1 reduces to the result in (4) in the linear case $\left(l_{i} \equiv 1\right)$. Despite its innocent appearance and fundamental nature, the proving of the result in Theorem 1 provides many interesting and subtle complexities.

Remark. The coefficients of recurrences attaining the maximal value given by Theorem 1 satisfy $\alpha_{x, y}=-1$ if $r(x)-r(y)$ is odd, and $\alpha_{x, y}=0$ otherwise. The same characteristic is satisfied for the posets considered in [3] (see Figure 1 ), for $\kappa$ odd or infinite, but not for $\kappa$ even. Hence, we pose the following open question.

Open Question 1 Suppose $\kappa$ is fixed, and $f$ is as in (2), where $\left\{\alpha_{u, v}\right\}$ satisfies (5). For what ranked posets $P$ is the maximal value of $|f(x)|$ for each $x \in P$ attained for $\left\{\alpha_{u, v}\right\}$ taken as $\alpha_{u, v}=-1$ for $r(u)-r(v)$ odd, and $\alpha_{u, v}=0$ otherwise?

For results showing that the answer to the question above is negative in the cases $\kappa=2,4$, in the linear case, see [7] and [4], respectively.

Applying results from a recent paper of the authors [8], along with Theorem 1, we may obtain immediate bounds for posets of the sort in (1) under certain constraints, such as fixed width and fixed number of elements. That is, suppose the sequence $\left\{y_{i}\right\}$ satisfies a general linear recurrence of the form

$$
y_{n}=a_{n} y_{n-1}+y_{n-2} \quad(n \geq 1),
$$

where $y_{0}=0, y_{1}=1$ and for each $i, a_{i}$ is a positive integer. Note that $y_{n}$ may be viewed as a function of $\left(a_{1}, a_{2}, \ldots, a_{n}\right)$, and hence we will denote $y_{n}$ by $y_{n}\left(a_{1}, a_{2}, \ldots, a_{n}\right)$.

In [8] the following two theorems regarding solutions to (8) were proven.

Theorem 2 Suppose that $\sum_{i=1}^{k} a_{i}=N$ then

$$
y_{k}\left(a_{1}, a_{2}, \ldots, a_{k}\right) \leq y_{k}(w, x, x, \ldots, x, w, w, \ldots, w)
$$

where $x=[N / k]$ and $w=x+1$. Here $[s]$ denotes the integer part of a real number s. Also note that the number of w's in (9) is precisely $N-k x$, while the number of $x$ 's is $k(x+1)-N$.

Theorem 3 Suppose that $\sum_{i=1}^{k+1} a_{i}=N+M$ and $\max a_{i}=M$, then

$$
y_{k+1}\left(a_{1}, a_{2}, \ldots, a_{k+1}\right) \leq y_{N+1}(M, 1,1, \ldots, 1) .
$$

Applying Theorems 1 and 2, we have 
Theorem 4 Suppose that $r(\hat{1})=k+1$ (i.e. the length of $P$ is $k+2$ ) and $\|P\|=N$. If $\kappa=\infty, f(\hat{0})=1$, and $\left\{\alpha_{u, v}\right\}$ satisfies (5), then for $x \in P$ with $r(x)=n,|f(x)| \leq y_{n}$, where $\left\{y_{i}\right\}$ is as given in the right hand side of (9) in the statement of Theorem 2 with $a_{j}=l_{j}$ for all $j$.

Similarly, employing Theorem 3, we have

Theorem 5 Suppose that the width of the poset $P$ is $M$ (i.e. $w(P)=\max \left\{l_{i}\right\}=$ $M)$ and $\|P\|=N$. If $\kappa=\infty, f(\hat{0})=1$, and $\left\{\alpha_{u, v}\right\}$ satisfies (5), then for $x \in P$ with $r(x)=n,|f(x)| \leq y_{n}$, where $\left\{y_{i}\right\}$ is as given in the right hand side of (10) in the statement of Theorem 3 with $a_{j}=l_{j}$ for all $j$.

Remark. (Möbius functions on $P$ ). An important example of a recursive sequence of the sort considered in (2) (in particular, those satisfying $\kappa=\infty$ and $\left\{\alpha_{u, v}\right\}$ as in (5)) is the Möbius function of $P$, i.e. the inverse of its Zeta function (cf. [22], [19], [14], [13]). In fact, let $\mathcal{I}(P)$ be the incidence algebra of $P$. Then, the inverse of any function $f \in \mathcal{I}(P)$, with $f(x, x)=1$ for all $x$, that takes values in $[0,1]$, would have coefficients as in (5). More broadly the set of functions being considered here are the inverses of reflexive anti-symmetric binary relations on $X$, the ground set of $P$ (c.f. Marenich [14], Stechkin [21], and Baranov and Stechkin [1]).

Remark. (Entries in inverses of triangular $\{0,1\}$ matrices) Some additional motivation for studying behavior of linear functions on $P$ with coefficients as in (5) stems from connections to triangular $\{0,1\}$ matrices with prescribed zero structures. In particular, suppose the elements in a poset $P$ as in (1) are enumerated successively via $\left\{x_{i}\right\}$ from $\hat{0}$, through the elements in $V_{1}$, then the elements in $V_{2}$, etc. The associated matrix for the Zeta function of $P$ has a prescribed zero in the $(i, j)^{t h}$ entry for $i \neq j$ whenever $x_{i}$ and $x_{j}$ are elements of the same antichain in $P$. Theorem 1 amounts to obtaining bounds on the entries in inverses of $\{0,1\}$ triangular matrices with prescribed zeroes in entries near the diagonal.

Example. Consider the class of $6 \times 6$ (unit diagonal) lower triangular, $\{0,1\}$ matrices, $\mathbf{L}=\left(l_{i, j}\right)$, satisfying the constraint $l_{3,2}=l_{5,4}=0$. Now, note that for $\mathbf{X}=\left(x_{i, j}\right)=\mathbf{L}^{-1}$, we have that $x_{1,1}=1$ and

$$
x_{i, 1}=\sum_{j=1}^{i-1}\left(-l_{i, j}\right) x_{j, 1}
$$

for $2 \leq i \leq 5$.

Employing Theorem 1, for the poset

$$
P=\hat{0} \oplus 2 \mathbf{1} \oplus 2 \mathbf{1} \oplus \hat{\mathbf{1}},
$$


we have that $\left|x_{2,1}\right| \leq B_{1},\left|x_{3,1}\right| \leq B_{1},\left|x_{4,1}\right| \leq B_{2},\left|x_{5,1}\right| \leq B_{2}$ and $\left|x_{6,1}\right| \leq B_{3}$, where $B_{1}=1, B_{2}=2$ and $B_{3}=5$.

The remainder of the paper proceeds as follows. Section 3 includes some preliminary lemmas and notation, while the proof of Theorem 1 is contained in Section 4.

\section{Preliminary Notation and Lemmas}

Let us write $\mathcal{P}=\{x \in P: f(x) \geq 0\}$ and $\mathcal{N}=\{x \in P: f(x)<0\}=\mathcal{P}^{c}$. This partitions the sign configuration of $\{f(x)\}_{x \in P}$. Now, define $g$ recursively via $g(0)=1$ and for $x$ with $r(x)=i$, with $i>1$,

$$
g(x)=-\sum_{\substack{y<x \\ y \in A^{c}}} g(y), \quad \text { for } \quad x \in A
$$

where $A$ is either $\mathcal{P}$ or $\mathcal{N}=\mathcal{P}^{c}$.

A simple induction with (13) shows that $g(y)$ and $f(y)$ have the same sign for all $y \in V$, and moreover

$$
|f(x)| \leq|g(x)|
$$

for all $x \in V$. Also note that for $x, y \in V_{i}$ with $f(x), f(y) \geq 0$ (resp. $f(x), f(y)<0)$, we have $g(x)=g(y)$. We denote this common value by $b_{+}(i)=g(x)$ (resp. $\left.b_{-}(i)=g(x)\right)$.

Much of the work in proving Theorem 1 will involve a comparison of coefficients in expansions of entries in the sequence $\left\{B_{i}\right\}$, as defined in $(7)$, with corresponding entries in the sequences $\left\{b_{+}(i)\right\}$ and $\left\{b_{-}(i)\right\}$. For that purpose, we will need the following technical lemma concerning $\left\{B_{i}\right\}$.

Lemma 1 Suppose that $\left\{B_{i}\right\}$ satisfies (7) with $B_{1}=1$ and $B_{j}=0$ for $j<1$. Then, for any $N>1$ and $1 \leq s \leq N-1$,

$$
B_{N}=\Theta_{N}(s) B_{N-s}+\Theta_{N}(s-1) B_{N-s-1},
$$

where $\Theta_{N}(0)=\Theta_{N}\left(0 ;\left\{l_{N-v}\right\}\right)=1$ and for $s \geq 1$,

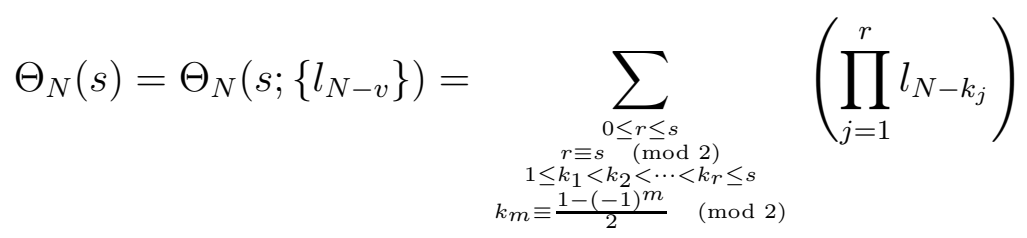

with the empty product (in the case of $r=0$ ) taken as 1 . 
Proof. The case $s=1$ follows directly from (7), since $\Theta_{N}(1)=l_{N-1}$ and $\Theta_{N}(0)=1$. Then, expanding via $(7)$, we have

$$
\begin{aligned}
B_{N} & =l_{N-1} B_{N-1}+B_{N-2}=l_{N-1}\left(l_{N-2} B_{N-2}+B_{N-3}\right)+B_{N-2} \\
& =\left(l_{N-1} l_{N-2}+1\right) B_{N-2}+l_{N-1} B_{N-3} \\
& =\Theta_{N}(2) B_{N-2}+\Theta_{N}(1) B_{N-3} .
\end{aligned}
$$

and the lemma holds for $s=2$. A simple induction now finishes the proof.

A more general version of our $\Theta$-function will be useful for proofs of other, more important results. For an arbitrary sequence $\left\{a_{j}\right\}_{j=1}^{i}$ we define

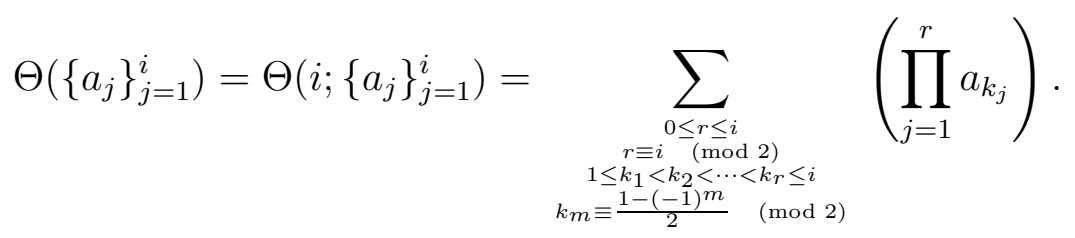

Note that the indices in each product term in (18) alternate between odd and even values, as can be seen in the next example.

Example. Note that evaluation of $\Theta\left(\left\{a_{j}\right\}\right)_{j=1}^{i}$ ) (as in (18)) for some small $i$ gives $\Theta\left(\left\{a_{1}\right\}\right)=a_{1}, \Theta\left(\left\{a_{1}, a_{2}\right\}\right)=1+a_{1} a_{2}, \Theta\left(\left\{a_{1}, a_{2}, a_{3}\right\}\right)=a_{1}+a_{3}+a_{1} a_{2} a_{3}$, and $\Theta\left(\left\{a_{1}, a_{2}, a_{3}, a_{4}\right\}\right)=1+a_{1} a_{2}+a_{1} a_{4}+a_{3} a_{4}+a_{1} a_{2} a_{3} a_{4}$.

Before coming to the proof of our main result, we prove the following simple inequality involving $\Theta$.

Lemma 2 For any $i \geq 1$, and positive real numbers, $a_{1}, a_{2}, \cdots, a_{i}$, we have

$$
\Theta\left(\left\{a_{j}\right\}_{j=1}^{i}\right) \geq \sum_{1 \leq j \leq i} a_{j}
$$

Proof. We consider two cases, depending on the parity of $i$ :

i. Case 1 ( $i$ odd). If $i=1$, then from (18) we have $\Theta\left(a_{1}\right)=a_{1}$, and the statement is true. Hence suppose $i \geq 3$. Then, considering the $r=1$ and $r=3$ terms in (18), and the fact that $a_{1}, a_{i}>0$, we have

$$
\begin{aligned}
\Theta\left(\left\{a_{j}\right\}_{j=1}^{i}\right) & \geq \sum_{\substack{1 \leq m \leq i \\
m \equiv 1}} a_{m}+a_{1}\left(\sum_{\substack{2 \leq n \leq i-1 \\
n \equiv 0}} a_{n}\right) a_{i} \\
& \geq \sum_{1 \leq j \leq i} a_{j} .
\end{aligned}
$$


ii. Case 2 ( $i$ even) The statement is obvious for $i=0$. So suppose $i \geq 2$. Considering the $r=0$ and $r=2$ terms in (18), and the fact that $a_{1}, a_{2}>0$, we have

$$
\begin{aligned}
& \Theta\left(\left\{a_{j}\right\}_{j=1}^{i}\right) \geq 1+a_{1} a_{2}+a_{1}\left(\sum_{\substack{4 \leq m \leq i \\
m \equiv 0}} a_{m}\right)+\left(\sum_{\substack{3 \leq n \leq i-1 \\
n \equiv 1}} a_{n}\right) a_{i} \\
& \geq\left(a_{1}+a_{2}\right)+\sum_{\substack{4 \leq m \leq i \\
m \equiv 0}} a_{m}+\sum_{\substack{3 \leq n \leq i-1 \\
n \equiv 1}} a_{n} \\
& =\sum_{1 \leq j \leq i} a_{j},
\end{aligned}
$$

the second inequality coming from $a_{1} a_{2}+1-\left(a_{1}+a_{2}\right)=\left(a_{1}-1\right)\left(a_{2}-1\right) \geq$ 0 .

We now turn to a proof of Theorem 1.

\section{Proof of Theorem 1}

In this section we prove Theorem 1.

The idea of the proof is straightforward. The key is to estimate the value of the function $|g(x)|$ from (13), and apply (14). The desired upper bound is obtained by explicitly describing the coefficients $\nabla(\cdot)$ of expressions for $b_{+}(N)$ and $b_{-}(N)$ (see $(27)$ ), and then comparing them with the coefficients $\Theta(\cdot)$ as in Lemma 1 , for a certain value of $s$ (see (28)). It turns out that the functions $\Theta(\cdot)$ serve as ideal upper bounds for the corresponding $\nabla(\cdot)$, when each is considered as a polynomial in $z_{i}=\left\|V_{i} \cap \mathcal{P}\right\|$. The process results favorably in effective bounds on $|g(x)|$.

Proof of Theorem 1. Recall that the sets $\mathcal{N}$ and $\mathcal{P}$ form a partition of $P$. We denote $\left|V_{i} \cap \mathcal{N}\right|$ via $v_{i}$ and $\left|V_{i} \cap \mathcal{P}\right|$ via $z_{i}$. Also, recall that $b_{+}(i)$ and $b_{-}(i)$ are defined as the values of $g(x)$, for $x \in V_{i} \cap \mathcal{P}$ and $x \in V_{i} \cap \mathcal{N}$, respectively.

Now, suppose that $\left\{B_{i}\right\}$ is as in (7), and that $\max \left\{\left|b_{+}(i)\right|,\left|b_{-}(i)\right|\right\} \leq B_{i}$, for $i<N$, and let

$$
I \stackrel{\text { def }}{=} \min \left\{i \geq 0: v_{N-i}=0\right\}
$$

Since $v_{1}=0$, by definition, $I$ is well defined.

To obtain recursive equations to compare with those established for $\left\{B_{i}\right\}$ in Lemma, we note that from (13),

$$
\begin{aligned}
b_{+}(N) & =\sum_{1 \leq j<N}-v_{j} b_{-}(j)=-v_{N-1} b_{-}(N-1)+\sum_{1 \leq j<N-1}-v_{j} b_{-}(j) \\
& =v_{N-1}\left|b_{-}(N-1)\right|+\left|b_{+}(N-1)\right| .
\end{aligned}
$$


Expanding further, via (13), we have

$$
\begin{aligned}
b_{+}(N) & =v_{N-1}\left(z_{N-2}\left|b_{+}(N-2)\right|+\left|b_{-}(N-2)\right|\right)+\left(v_{N-2}\left|b_{-}(N-2)\right|+\left|b_{+}(N-2)\right|\right) \\
& =\left(v_{N-1} z_{N-2}+1\right) b_{+}(N-2)+\left(v_{N-1}+v_{N-2}\right) b_{-}(N-2),
\end{aligned}
$$

and more generally,

$$
b_{+}(N)=\nabla_{1}(N, s)\left|b_{-}(N-j)\right|+\nabla_{0}(N, s)\left|b_{+}(N-j)\right|,
$$

where, for $e=0,1$ the function $\nabla_{e}(N, s)$ is defined as

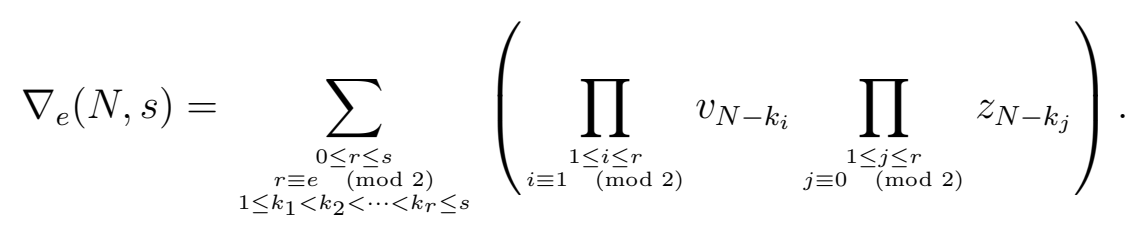

Note that in the case of the $\nabla_{e}$, the factors in the product terms vary between entries in $\left\{v_{i}\right\}$ and ones in $\left\{z_{i}\right\}$, as is indicated in the next example.

Example. Note that for fixed $\left(v_{N-i}, z_{N-i}\right)=\left(\bar{v}_{i}, \bar{z}_{i}\right), 1 \leq i \leq 4$, evaluation of $\nabla_{e}(N, 4)$ (as in $\left.(26)\right)$ for $e=0,1$ gives

$$
\begin{aligned}
\nabla_{0}(N, 4) & =1+\bar{v}_{1} \bar{z}_{2}+\bar{v}_{1} \bar{z}_{3}+\bar{v}_{1} \bar{z}_{4}+\bar{v}_{2} \bar{z}_{3}+\bar{v}_{2} \bar{z}_{4}+\bar{v}_{3} \bar{z}_{4}+\bar{v}_{1} \bar{z}_{2} \bar{v}_{3} \bar{z}_{4} \\
\nabla_{1}(N, 4) & =\bar{v}_{1}+\bar{v}_{2}+\bar{v}_{3}+\bar{v}_{4}+\bar{v}_{1} \bar{z}_{2} \bar{v}_{3}+\bar{v}_{1} \bar{z}_{2} \bar{v}_{4}+\bar{v}_{1} \bar{z}_{3} \bar{v}_{4}+\bar{v}_{2} \bar{z}_{3} \bar{v}_{4}
\end{aligned}
$$

Since $v_{N-I}=0$, we have $\left|b_{+}(N-I+1)\right|=\left|b_{+}(N-I)\right|$, and hence (25) with the induction hypothesis gives

$$
\begin{aligned}
\left|b_{+}(N)\right| & =\nabla_{1}(N, I-1)\left|b_{-}(N-I+1)\right|+\nabla_{0}(N, I-1)\left|b_{+}(N-I)\right| \\
& \leq \nabla_{1}(N, I-1) B_{N-I+1}+\nabla_{0}(N, I-1) B_{N-I}
\end{aligned}
$$

On the other hand, Equation (15), with $s=I-1$ gives

$$
B_{N}=\Theta_{N}(I-1) B_{N-I+1}+\Theta_{N}(I-2) B_{N-I} .
$$

Hence, in obtaining $\left|b_{+}(N)\right| \leq B_{N}$, we need only compare (27) and (28). To that end, for convenience, we introduce the notation

$$
\Theta(i, j) \stackrel{\text { def }}{=} \begin{cases}\Theta\left(\left\{l_{N-i}, \cdots, l_{N-j}\right\}\right), & \text { if } i \leq j \\ 1, & \text { otherwise. }\end{cases}
$$

Then, considering $\left\{\nabla_{1}(N, s)\right\},\left\{\nabla_{0}(N, s)\right\}$ and $\left\{\Theta\left(\left\{l_{N-m}\right\}_{m=1}^{s}\right)\right\}$ as polynomials in variables $\left(z_{N-1}, z_{N-2}, \cdots, z_{N-s}\right.$ ) (and recalling that $l_{j}=z_{j}+v_{j}$ for all 
$j$ ), we obtain that for $r>0$ and $1 \leq k_{1}<k_{2}<\cdots<k_{r} \leq s$ (with $k_{i}-k_{i-1}>1$ for $1<i \leq r$, if $r>1$ ), the coefficients of $\widetilde{z} \stackrel{\text { def }}{=} z_{N-k_{1}} z_{N-k_{2}} \cdots z_{N-k_{r}}$ in $\nabla_{1}(N, s)$ and $\nabla_{0}(N, s)$, are given by

$$
[\widetilde{z}] \nabla_{1}(N, s)= \begin{cases}\prod_{m=1}^{r+1}\left(\sum_{i=k_{m-1}+1}^{k_{m}-1} v_{N-i}\right), & \text { if } k_{1}>1 \text { and } k_{r}<s \\ 0 & \text { otherwise }\end{cases}
$$

where we define $k_{0}=0$ and $k_{r+1}=s+1$. Also

$$
[\widetilde{z}] \nabla_{0}(N, s)= \begin{cases}\prod_{m=1}^{r}\left(\sum_{i=k_{m-1}+1}^{k_{m}-1} v_{N-i}\right), & \text { if } k_{1}>1 \\ 0 & \text { otherwise }\end{cases}
$$

where we again define $k_{0}=0$.

Example. Note that, referring to the last example, we have $\nabla_{0}(N, 4)=$ $1+\left(\bar{v}_{1}\right) \bar{z}_{2}+\left(\bar{v}_{1}+\bar{v}_{2}\right) \bar{z}_{3}+\left(\bar{v}_{1} \bar{v}_{3}\right) \bar{z}_{2} \bar{z}_{4}$ and $\nabla_{1}(N, 4)=\left(\bar{v}_{1}+\bar{v}_{2}+\bar{v}_{3}+\bar{v}_{4}\right)+\bar{v}_{1}\left(\bar{v}_{3}+\right.$ $\left.\bar{v}_{4}\right) \bar{z}_{2}+\left(\bar{v}_{1}+\bar{v}_{2}\right) \bar{z}_{3}$.

Now, in a very similar way, we can compute the coefficients of $\widetilde{z}$ in $\Theta\left(\left\{l_{N-m}\right\}_{m=1}^{s}\right)$ and $\Theta\left(\left\{l_{N-m}\right\}_{m=1}^{s-1}\right)$. We have

$$
[\widetilde{z}] \Theta\left(\left\{l_{N-m}\right\}_{m=1}^{s}\right)= \begin{cases}\prod_{j=1}^{r+1} \Theta\left(k_{j-1}+1, k_{j}-1\right), & \text { if } k_{r}<s \\ \prod_{j=1}^{r} \Theta\left(k_{j-1}+1, k_{j}-1\right), & \text { if } k_{r}=s\end{cases}
$$

where $k_{0}=0$ and $k_{r+1}=s+1$. Similarly, changing $s$ to $s-1$, we obtain

$$
[\widetilde{z}] \Theta\left(\left\{l_{N-m}\right\}_{m=1}^{s-1}\right)= \begin{cases}\prod_{j=1}^{r} \Theta\left(k_{j-1}+1, k_{j}-1\right), & \text { if } k_{r}<s-1 \\ \prod_{j=1}^{r-1} \Theta\left(k_{j-1}+1, k_{j}-1\right), & \text { if } k_{r}=s-1 \\ 0 & \text { otherwise }\end{cases}
$$

with $k_{0}=0$ and $k_{r+1}=s$.

Here let us note that the constant terms (i.e. those free of $z$ ) in the expansions of $\nabla_{1}(N, s), \nabla_{0}(N, s), \Theta\left(s,\left\{l_{N-m}\right\}_{m=1}^{s}\right)$ and $\Theta\left(\left\{l_{N-m}\right\}_{m=1}^{s-1}\right)$ are $\sum_{i=1}^{s} v_{N-i}, 1, \Theta(1, s)$ and $\Theta(1, s-1)$, respectively.

Combining this with Lemma 2 and (30)-(33) gives the following result. 
Lemma 3 We have

1. If $k_{r}<i$, then

$$
[\widetilde{z}] \Theta\left(\left\{l_{N-m}\right\}_{m=1}^{s}\right) \geq[\widetilde{z}] \nabla_{1}(N, s) \text { and }[\widetilde{z}] \Theta\left(\left\{l_{N-m}\right\}_{m=1}^{s-1}\right) \geq[\widetilde{z}] \nabla_{0}(N, s) \text {. }
$$

2. If $k_{r}=s$, then

$$
[\widetilde{z}] \Theta\left(\left\{l_{N-m}\right\}_{m=1}^{s}\right) \geq[\widetilde{z}] \nabla_{1}(N, s)=0=[\widetilde{z}] \nabla_{0}(N, s)=[\widetilde{z}] \Theta\left(\left\{l_{N-m}\right\}_{m=1}^{s-1}\right)
$$

\section{Moreover}

$$
\text { [1] } \Theta\left(\left\{l_{N-m}\right\}_{m=1}^{s}\right) \geq[1] \nabla_{1}(N, s) \text { and }[1] \Theta\left(\left\{l_{N-m}\right\}_{m=1}^{s-1}\right) \geq[1] \nabla_{0}(N, s) \text {. }
$$

By necessity, the sequence $\left\{B_{i}\right\}$ is non-decreasing, and hence, the results for $b_{+}(N)$ follow upon employing Lemma 3, (28) and (27). In particular, when $k_{r}=s=I-1$, we have, by Lemma $3(2)$,

$$
\begin{aligned}
([\widetilde{z}] & \left.\nabla_{1}(N, I-1)\right) B_{N-I+1}+\left([\widetilde{z}] \nabla_{0}(N, I-1)\right) B_{N-I} \\
= & \left([\widetilde{z}] \nabla_{0}(N, I-1)\right) B_{N-I} \leq\left([\bar{z}] \Theta_{N}(I)\right) B_{N-I} \leq\left([\bar{z}] \Theta_{N}(I)\right) B_{N-I+1} \\
& =\left([\bar{z}] \Theta_{N}(I-1)\right) B_{N-I+1}+\left([\bar{z}] \Theta_{N}(I-2)\right) B_{N-I}
\end{aligned}
$$

By Lemma 3(3),

$$
\begin{aligned}
([1] & \left.\nabla_{1}(N, I-1)\right) B_{N-I+1}+\left([1] \nabla_{0}(N, I-1)\right) B_{N-I} \\
& \leq\left([1] \Theta_{N}(I-1)\right) B_{N-I+1}+\left([1] \Theta_{N}(I-2)\right) B_{N-I},
\end{aligned}
$$

and similarly, by Lemma $3(1)$, if $k_{r}<s=I-1$,

$$
\begin{aligned}
([\widetilde{z}] & \left.\nabla_{1}(N, I-1)\right) B_{N-I+1}+\left([\widetilde{z}] \nabla_{0}(N, I-1)\right) B_{N-I} \\
\leq & \left([\bar{z}] \Theta_{N}(I-1)\right) B_{N-I+1}+\left([\bar{z}] \Theta_{N}(I-2)\right) B_{N-I}
\end{aligned}
$$

Combining (34)-(36) with (27) and (28), we have $\left|b_{+}(N)\right| \leq B_{N}$, as required.

A similar argument works when considering $b_{-}(N)$ in place of $b_{+}(N)$. Here, in place of $(22)$, we define $I^{*} \stackrel{\text { def }}{=} \min \left\{i>0: z_{N-i}=0\right\}$, and proceed as before. Note that while $z_{1}=\left\|\mathcal{P} \cap V_{1}\right\|$ may not be zero, since $f(\hat{0})=1$, we have for $x \in \mathcal{P} \cap V_{1}, f(x)=0$ and hence for the purpose of maximizing $|f(y)|$, for $y \in P$ with $r(y)>1$, we may assume that $z_{1}=0$, which guarantees the existence of $I^{*}$. 


\section{References}

[1] V. I. Baranov And B. S. Stechkin. Extremal combinatorial problems and their applications, Dordrecht, Boston, Kluwer Academic Publishers, (1995).

[2] K. S. Berenhaut and J. D. Foley. Finite Order Linear Recurrences and an Application to $(0,1)$ Matrices, preprint, submitted (2005), 19 pp.

[3] K. S. Berenhaut And J. D. Foley Explicit Bounds for Multidimensional Linear Recurrences with Restricted Coefficients, Journal of Mathematical Analysis and Applications, Volume 322, Issue 2, (2006), 1159-1167.

[4] K. S. Berenhaut, B. G. Gibson, J. H. Newman and J. F. AnDERSON. Bounds for fourth order $(0,1)$ difference equations, In press, Computers and Mathematics with Applications (2006).

[5] K. S. Berenhaut And R. B. Lund. Bounds for linear recurrences with restricted coefficients, Journal of Inequalities in Pure and Applied Mathematics 4, 2, Article 26 (2003), 15 pp.

[6] K. S. Berenhaut and D. C. Morton. Second-order bounds for linear recurrences with negative coefficients, Journal of Computational and Applied Mathematics, 186 (2006), 504-522.

[7] K. S. Berenhaut, D. C. Morton and Y. W. Fan. Bounds for second order recurrences in terms of maximal products over integer partitions, Proceedings of the Eleventh International Conference on Fibonacci Numbers, to Appear (2004), 14 pp.

[8] K. S. Berenhaut, A. B. O’Keefe And F. Saidak. Recursive sequences of the form $y_{n}=a_{n} y_{n-1}+y_{n-2}$ with integer coefficients, Indian Journal of Mathematics, Vol. 48, no. 1 (2006), 39-54.

[9] G. Everest, A. van der Poorten, I. Shparlinski and T. WARD. Recurrence sequences. Mathematical Surveys and Monographs, 104. American Mathematical Society, Providence, RI, (2003), xiv+318 pp.

[10] L. Flatto, J. C. Lagarias, And B. Poonen. Lap numbers and periodic points of the beta transformation, Ergodic Theory and Dynamical Systems, (2) 14 (1994), 237-266.

[11] R. L. Graham and N. J. A. Sloane. Anti-Hadamard Matrices, Linear Algebra and Applications, 62 (1984), 113-137. 
[12] A. Odlyzko, And B. Poonen. Zeros of polynomials with 0,1 coefficients, L'Enseignement Mathematique, 39 (1993), 317-348.

[13] D. N. Kozlov. Maximizing the Möbius function of a poset and the sum of the Betti numbers of the order complex. Combinatorica 19 (1999), no. 4, 533-548.

[14] E. E. Marenich. Bound for values of the Möbius function, Mat. Zametki 44 (1988), no. 4, 469-487.

[15] R. Pemantle and M. C. Wilson. Asymptotics of multivariate sequences I: smooth points of the singular variety, Journal of Combinatorial Theory A 97 (2002), 129-161.

[16] R. Pemantle and M. C. Wilson. Asymptotics of multivariate sequences II: multiple points of the singular variety Combinatorics, Probability and Computing 13 (2004), no. 4-5, 735-761.

[17] M. Petkovšek And M. Bousquet-MÉlou. Linear recurrences with constant coefficients: the multivariate case. Discrete Mathematics, 225 (2000), no. 1-3, 51-75.

[18] K. H. Rosen (Ed.) Handbook of Discrete and Combinatorial Mathematics. Boca Raton, FL: CRC Press, (2000).

[19] B. E. Sagan, Y. N. Yeh And G. M. Ziegler. Maximizing Möbius functions on subsets of Boolean algebras. Discrete Math. 126 (1994), no. $1-3,293-311$.

[20] B. Solomyak. Conjugates of beta-numbers and the zero-free domain for a class of analytic functions, Proceedings of the London Mathematical Society, 68 (1994), 477-498.

[21] B. S. Stechkin. Binary functions on ordered sets, Trudy Mat. Inst. Akad. Nauk., 143 (MIAN) SSSR, 178-187.

[22] G. M. Ziegler. Posets with maximal Möbius function. J. Combin. Theory Ser. A 56 (1991), no. 2, 203-222.

second-order 439-448.

\section{Received: December 7, 2006}

